Endocr Metab Immune Disord Drug Targets 2021;21(5):956-965.

\title{
What is the Impact of Cinnamon Supplementation on Blood Pressure? A Systematic Review and Meta-Analysis
}

\author{
Abed Ghavami' ${ }^{1}$, Hossein K Haghighian ${ }^{2}$, Neda Roshanravan ${ }^{3}$, Rahele Ziaei ${ }^{1}$, Ehsan \\ Ghaedi $^{4}$, Amir R Moravejolahkami ${ }^{1}$, Gholamreza Askari ${ }^{5}$ \\ ${ }^{1}$ Student Research Committee, Department of Clinical Nutrition, School of Nutrition and Food \\ Science, Isfahan University of Medical Sciences, Isfahan, Iran. \\ ${ }^{2}$ Department of Nutrition, School of Health, Qazvin University of Medical Sciences, Qazvin, \\ Iran. \\ ${ }^{3}$ Cardiovascular Research Center, Tabriz University of Medical Sciences, Tabriz, Iran. \\ ${ }^{4}$ Students' Scientific Research Center (SSRC), Tehran University of Medical Sciences (TUMS), \\ Tehran, Iran. \\ ${ }^{5}$ Food Security Research Center, Department of Community Nutrition, School of Nutrition and \\ Food Science, Isfahan University of Medical Sciences, Isfahan, Iran.
}

DOI: $\underline{10.2174 / 1871530320666200729143614}$

\begin{abstract}
Aim: This study was designed to make a systematic review and meta-analysis on randomized controlled trials (RCTs) assessing the effect of cinnamon on blood pressure (BP).

Methods: A systematic computerized literature search of PubMed, Scopus, Web of Science, Cochrane Library and Google Scholar databases was conducted up to August 2019. All RCTs using cinnamon supplements in adults were included in this systematic review and metaanalysis.
\end{abstract}

Results: Out of 927 records, 8 trials that enrolled 582 participants were included. The pooled effect size showed that SBP did not change following cinnamon supplementation. (WMD: $0.61 \mathrm{mmHg}$; 95\% CI: $-1.36,0.14, \mathrm{P}=0.111)$. Also cinnamon supplementation in long-duration ( $\geq$ 8weeks) had a significant effect on SBP (WMD: $-1.25 \mathrm{mmHg}$; 95\% CI: -2.22, -0.28, P=0.012). Pooled analysis showed that cinnamon had a significant effect on DBP (WMD: $-0.93 \mathrm{mmHg}$, $95 \% \mathrm{CI}:-1.55$ to $-0.32, \mathrm{P}=0.003)$. In addition, results from both duration subsets and high dose (>1500 mg/day) of cinnamon supplementation were significant.

Conclusion: Our findings revealed that cinnamon supplementation has favorable effects on DBP although results of SBP were not the same. Nonetheless, further studies are required.

Keywords: Blood pressure; cardiovascular disease; cinnamon; herbal medicine; meta-analysis; systematic review. 